\title{
Impairment of lysosomal integrity by B10, a glycosylated derivative of betulinic acid, leads to lysosomal cell death and converts autophagy into a detrimental process
}

\author{
P Gonzalez ${ }^{1}$, I Mader ${ }^{1}$, A Tchoghandjian ${ }^{2}$, S Enzenmüller ${ }^{1}$, S Cristofanon ${ }^{2}$, F Basit ${ }^{2}$, K-M Debatin ${ }^{1}$ and S Fulda ${ }^{\star, 1,2}$
}

In this study, we report a novel mechanism of action for a cytotoxic derivative of betulinic acid (BA). B10 is a semi-synthetic glycosylated derivative of BA selected for its enhanced cytotoxic activity. Interestingly, although B10 induces apoptosis, caspase-3 downregulation incompletely prevents B10-induced cell death, Bcl-2 overexpression fails to protect cells and DNA fragmentation rates do not reflect cell death rates in contrast to cytoplasmic membrane permeabilization. These results implicate that apoptotic and non-apoptotic cell death coexist upon B10 treatment. Unexpectedly, we found that B10 induces autophagy and also abrogates the autophagic flux. B10 destabilizes lysosomes as shown by Lysotracker Red staining and by cathepsin $Z$ and $B$ release from lysosomes into the cytoplasm. Consistently, the cathepsin inhibitor Ca074Me significantly decreases B10-induced cell death, further supporting the fact that the release of lysosomal enzymes contributes to B10-triggered cell death. Downregulation of ATG7, ATG5 or BECN1 by RNAi significantly decreases caspase-3 activation, lysosomal permeabilization and cell death. Thus, by concomitant induction of autophagy and inhibition of the autophagic flux, B10 turns autophagy into a cell death mechanism. These findings have important implications for the therapeutic exploitation of BA derivatives, particularly in apoptosis-resistant cancers.

Cell Death and Differentiation (2012) 19, 1337-1346; doi:10.1038/cdd.2012.10; published online 17 February 2012

Bioactive natural compounds or their semi-synthetic derivatives offer a considerable therapeutic potential against cancer. ${ }^{1}$ Among these, betulinic acid (BA), a triterpenoid initially derived from white birch tree, has received attention because of its multiple biological activities in mammalian cells. The pluripotency of BA is of great interest, as this compound is active against HIV, parasitic diseases and cancer. ${ }^{2-5}$ Nevertheless, the therapeutic application of this drug is currently limited because of its poor solubility in aqueous solvents and an ill-defined mode of action. To overcome these limitations, a wide variety of semi-synthetic derivatives of BA has been generated to improve its pharmacological properties for in vivo studies. In addition, detailed studies are being performed to elucidate the mechanisms of action underlying their cytotoxic activity. To date, BA and its derivatives have been reported to induce cell death in cancer cells via the activation of the intrinsic pathway of apoptosis. ${ }^{6}$ Accordingly, BA triggers the release of pro-apoptotic factors, such as cytochrome- $c$, from the mitochondria supposedly by interacting with the permeability pore transition complex and by modifying the balance of pro- and anti-apoptotic proteins of the Bcl-2 protein family. ${ }^{6}$ In addition, BA and its derivatives appear to interfere with multiple signaling pathways involved in survival. PI3K, NF- $\kappa \mathrm{B}$ and lipid metabolism are modulated by BA-related compounds, but whether and how these effects contribute to cell death is unknown. ${ }^{7-10} \mathrm{~A}$ better understanding of the mechanism of action of these compounds is critical to promote their therapeutic usage. Therefore, we investigated in the present study the mode of action of $\mathrm{B} 10, \mathrm{a}$ new glycosylated derivative of BA presenting enhanced cytotoxic activity. We show that B10 triggers a mixed phenotype of cell death characterized by morphological and biochemical features of apoptotic and non-apoptotic cell death. This complex mode of cell death previously unreported for BA and its derivatives is related to induction of autophagy and to the disruption of the autophagic flux, which contributes to the intracytoplasmic release of lysosomal enzymes. Our findings refine the conception of the mechanism of action of BA-derived triterpenoids. These novel inputs might influence the therapeutic application of BA-related compounds.

\section{Results}

B10 triggers non-apoptotic forms of cell death. First, we evaluated the effect of B10 on a panel of glioblastoma cell lines. B10 induced a profound loss of viability in all cell lines

\footnotetext{
${ }^{1}$ University Children's Hospital, Ulm University, Eythstrasse 24, 89075 Ulm, Germany and ${ }^{2}$ Institute for Experimental Cancer Research in Pediatrics, Goethe-University Frankfurt, Komturstr. 3a, 60528 Frankfurt am Main, Germany

${ }^{*}$ Corresponding author: S Fulda, Institute for Experimental Cancer Research in Pediatrics, Goethe-University Frankfurt, Komturstr. 3a, 60528 Frankfurt am Main, Germany. Tel: + 4969 67866557; Fax: + 4969 6786659157; E-mail: simone.fulda@kgu.de

Keywords: betulinic acid; apoptosis; autophagy; lysosomes

Abbreviations: ATG, autophagy-related genes; BA, betulinic acid; CHX, cycloheximide; CTSZ, cathepsin Z; LTR, lysotracker red; KD, knock-down; MMP, mitochondrial membrane potential; MTT, 3-(4,5-dimethylthiazol-2-yl)-2,5-diphenyltretrazolium bromide; PI, propidium iodide; PI3K, phosphoinositide 3-kinase; siRNA, small interfering RNA; TMRM, tetramethylrhodamine methyl ester perchlorate

Received 28.9.10; revised 24.12.11; accepted 29.12.11; Edited by P Mehlen; published online 17.2.12
} 
A
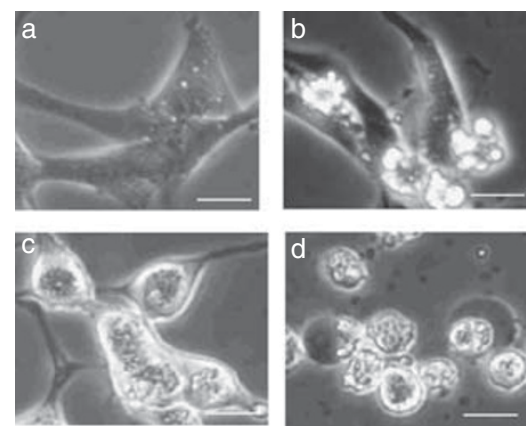

C $801 \square \mathrm{A} 5+\mathrm{PI}$

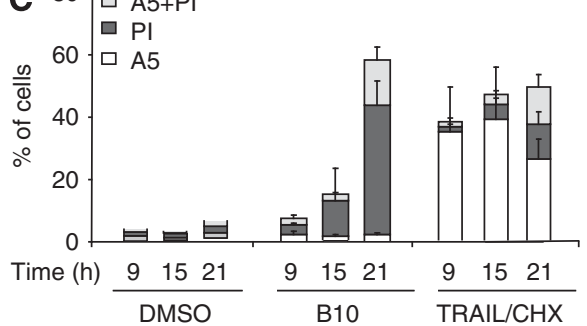

D
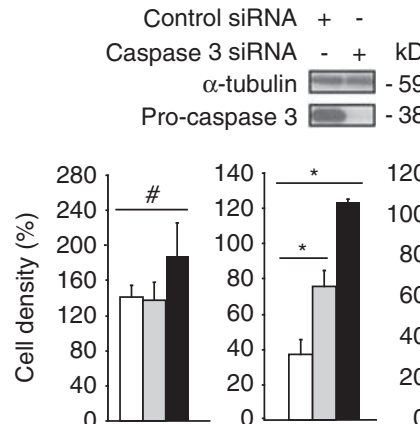

Control siRNA + - -

Caspase 3 siRNA - + -

zVAD.fmk - - +

DMSO

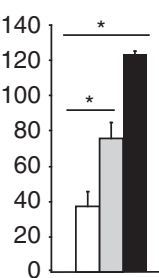

$+-$

$-+$

$-\cdot+$

B10
B

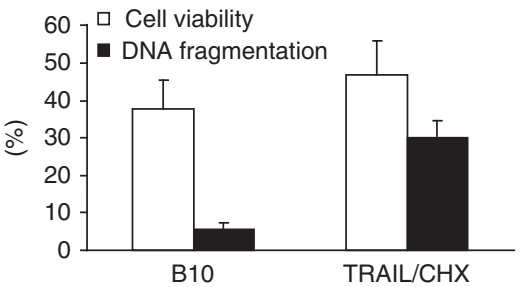

E

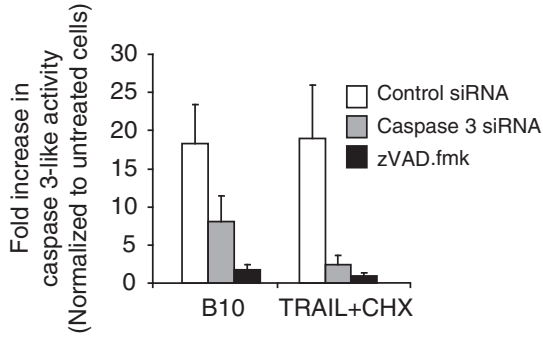

$+-$

- +

- - +

TRAIL+CHX

Figure 1 B10-induced cell death is partially apoptotic. (A) Morphological alterations of B10-treated U87MG cells were analyzed using microscopy. Representative pictures of the cell death phenotype of untreated cells (a), cells treated with TRAIL $(20 \mathrm{ng} / \mathrm{ml})$ and CHX (1 $\mu \mathrm{g} / \mathrm{ml})$ (b) or cells exposed to B10 (18 $\mu \mathrm{M})$ for $9 \mathrm{~h}$ (c) or $21 \mathrm{~h}$ (d) are shown (scale bar: $30 \mu \mathrm{m}$ ). The combination treatment with TRAIL and CHX was used as a positive control for apoptotic morphology. (B) U87MG cells treated with B10 (18 $\mu \mathrm{M})$ or TRAIL/CHX were analyzed for loss of cell viability by determining cell density via crystal violet staining, and DNA fragmentation by analysis of sub-G1 DNA content ( $n=3$, mean + S.D.). (C) The exposure of phosphatidylserine was analyzed by annexin V staining in parallel to membrane permeabilization assessed by PI in B10- or TRAIL/CHXtreated cells. Mean + S.D. of three independent experiments are shown. (D) The cytotoxic activity of B10 was evaluated using crystal violet staining in U87MG with downregulated pro-caspase- 3 or in the presence of $40 \mu \mathrm{M}$ zVAD.fmk. Mean + S.D. of three independent experiments are shown, ${ }^{*} P<0.02$, \#P>0.05. TRAIL/CHX combination treatment was used as a positive control for apoptosis inhibition upon pro-caspase-3 downregulation. (E) Caspase-3-like activity was measured in cells transfected with control or caspase-3-specific siRNA incubated with B10 $(21 \mathrm{~h})$ or TRAIL/CHX (15 h) using the fluorogenic substrate zDEVD-R110 that measures caspase-3 and other DEVD-specific protease activities

(Supplementary Figure S1). Interestingly, the morphology of dying cells was rarely typically apoptotic, suggesting that the cytotoxic activity of B10 is also mediated by non-apoptotic modes of cell death. In order to investigate this assumption in detail, the well-characterized U87MG cell line was selected for further studies. Analysis of morphological alterations by microscopy revealed a mixed phenotype of dying cells among the cell population over time. Although a minor subpopulation of U87MG cells clearly displayed typical apoptotic blebbing, which is also observed in these cells upon treatment with TRAIL and cycloheximide ( $\mathrm{CHX})$ used as a positive control for apoptotic cell death, ${ }^{11}$ the majority of cells treated with B10 did not present intense membrane blebbing (Figure 1Ab). Instead, spherical and swollen cells were observed during an intermediate phase of cell death (Figure 1Ac). At a late phase, these cells ended up with a terminal bleb, characterized by a dense aggregate of the intracytoplasmic material segregated into a rounded cytoplasmic membrane before detaching from the substrate (Figure 1Ad). This morphology is usually not found during apoptosis and therefore suggests the occurrence of a non-apoptotic mode of cell death.

In line with this notion, the amount of DNA fragmentation, a characteristic feature of apoptosis, did not correspond to the 
death rates determined by changes in cell density (Figure 1B). In contrast, TRAIL/CHX-induced loss of viability was accompanied by a concomitant increase in DNA fragmentation (Figure 1B). In addition, annexin V/propidium iodide (PI) staining revealed that treatment with $\mathrm{B} 10$ predominately caused an increase in PI-positive cells, whereas treatment with TRAIL/CHX resulted in an increase in Annexin $V$ positive cells (Figure $1 \mathrm{C}$ ). Further, overexpression of $\mathrm{Bcl}-2$ did not prevent B10-induced cell death or caspase-3 activation, whereas $\mathrm{Bcl}-2$ protected against cell death and caspase-3 activation upon treatment with TRAIL (Supplementary Figure S2). To determine whether caspase-3 activity is necessary for B10-induced cell death, caspase-3 expression was downregulated via RNA interference. Knockdown of caspase-3 resulted in a significant, yet incomplete decrease in B10induced caspase-3-like activity and cytotoxicity (Figures 1D and E), pointing to a partial involvement of caspase-3 in B10induced cell death. In comparison, caspase-3-silencing almost completely protected against TRAIL/CHX-induced caspase-3-like activity and cell death (Figures 1D and E). Noteworthy, the addition of zVAD.fmk provided a better protection against B10-induced caspase-3-like activity and cell death than caspase-3 downregulation (Figures 1D and E). Besides residual caspase-3 expression upon RNAi-mediated silencing, this difference may be attributed to the inhibition of additional proteases unrelated to caspases but sensitive to zVAD.fmk, ${ }^{12,13}$ which might also contribute to caspase-3-like activity and cell death upon B10 treatment. In comparison, caspase-3-silencing was nearly as effective as zVAD.fmk in preventing TRAIL/CHX-induced caspase-3-like activity and cell death (Figures 1D and E). Together, these results indicate that B10 triggers a mixed type of cell death with apoptotic and non-apoptotic features compared with the prototypic apoptotic stimulus TRAIL. This prompted us to investigate the alternative mechanisms of action for B10.

B10 treatment destabilizes Iysosomes. Analysis of B10treated cells using electron microscopy revealed a marked cytoplasmic accumulation of vesicles (Figure 2A). Because this points to a possible alteration of lysosomal/endosomal trafficking, we investigated its implication in B10-induced cell death. Firstly, to evaluate the integrity of the lysosomal compartment, we used LysoTracker Red (LTR), a dye that accumulates in acidic compartments. Interestingly, the appearance of a subpopulation of cells presenting a high LTR fluorescence within $9 \mathrm{~h}$ of treatment pointed to an increased volume of the acidic compartment in B10-treated cells (Figure 2B). However, this increase was transient as it was not detectable at later time points (Figure 2B). Conversely, a population of low LTR fluorescence increased from $15 \mathrm{~h}$ onwards, indicating that the volume of the acidic compartment decreases over time in B10-treated cells (Figure 2B). To further determine whether the reduction of the volume of the acidic compartment upon B10 treatment was due to lysosomal permeabilization, the localization of cathepsin Z (CTSZ) and cathepsin B (CTSB), two lysosomal enzymes, was analyzed by immunofluorescence. Although CTSZ was found in the lysosomes of untreated cells, the enzyme was detected diffusely in the cytoplasm and in the nucleus upon B10 treatment (Figure 2C). In addition, separation into lysosomal and cytoplasmic fractions revealed that treatment with B10 caused a decrease of CTSZ and CTSB in the lysosomal fraction and their concomitant increase in the cytoplasmic fraction (Figures 2D and $E$ ). This altered distribution of CTSZ and CTSB therefore confirms the permeabilization of lysosomal membranes upon B10 treatment.

Cathepsins are implicated in B10-induced cell death by apoptotic and non-apoptotic cell death. As B10 induces Iysosomal permeabilization (Figure 2), the role of lysosomal enzymes in B10-induced cell death was investigated using chemical inhibitors of lysosomal enzymes. As shown in Figure 3A, Ca074Me, an inhibitor of CTSB, L, and S, significantly delayed cell death in U87MG, whereas neither E64 nor leupeptin prevented B10-induced cell death (Figure 3A). Cathepsins can cleave a number of substrates upon their release into the cytoplasm. To explore possible substrates of the lysosomal enzymes inhibited by CA074Me, we focussed on some components of the apoptotic machinery. ${ }^{14}$ We found that $\mathrm{Ca} 074 \mathrm{Me}$ prevented the $\mathrm{B} 10-$ induced decrease of Bid protein expression (Figure $3 \mathrm{Ba}$ ), indicating that Bid may be degraded by cathepsins that are inhibited by CA074Me. However, knockdown of Bid did not protect against B10-induced cell death, whereas it significantly inhibited TRAIL/CHX-triggered cell death (data not shown). Ca074Me delayed MMP loss upon B10 exposure and transiently reduced caspase- 3 activation at $15 \mathrm{~h}$, whereas no difference in caspase-3 cleavage was observed at a later time point (Figures $3 \mathrm{Bb}$ and $\mathrm{C}$ ). Noteworthy, this transient inhibitory effect of $\mathrm{Ca} 074 \mathrm{Me}$ on caspase-3 activation did not reflect the 24-h delay of cell death upon treatment with this inhibitor (Figure $3 \mathrm{~A}$ ). This set of experiments suggests that lysosomal enzymes are involved both in apoptotic and nonapoptotic cell death in B10-treated cells.

B10 induces autophagy and disrupts the autophagic flux. As B10 impairs the integrity of the lysosomal compartment and because autophagy relies on lysosomes for the degradation of autophagosomal cargos to promote cell fitness, we investigated the effects of B10 on autophagy using LC3 as a marker of autophagic activity. Remarkably, LC3 lipidation (LC3-II) increased upon B10 treatment in a timedependent manner (Figure 4A) and this marker translocated to autophagosomes represented by vesicular structures containing LC3 (Figure 4B). These results indicate that B10 induces autophagy and/or blocks the autophagic flux. To investigate possible causes for autophagy induction by B10, we analyzed the activation status of the PI3K/Akt/mTOR pathway in B10-treated cells, as this pathway is a key regulator of autophagy. PI3K/Akt/mTOR signaling was progressively inhibited upon treatment with $\mathrm{B} 10$ as indicated by decreased phosphorylation of Akt, a downstream target of $\mathrm{PI} 3 \mathrm{~K}$, as well as decreased phosphorylation of S6 and 4EBP1, both downstream targets of mTOR (Figure 4C). Moreover, the kinetic of inactivation of PI3K/Akt/mTOR signaling occurred in parallel with the induction of autophagy as assessed by GFP-LC3 localization to autophagosomes (Figure 4Bb). To further determine the role of PI3K/ Akt/mTOR signaling in B10-triggered autophagy, we used $\alpha$-tocopherol to prevent the B10-mediated inhibition of the 
PI3K/Akt/mTOR pathway, as $\alpha$-tocopherol has previously been reported to stabilize lipid rafts, thereby stimulating PI3K activity and thus PI3K/Akt/mTOR signaling. ${ }^{15}$ Addition of $\alpha$-tocopherol partly preserved phosphorylation of Akt and S6, reduced LC3 lipidation upon B10 treatment and significantly reduced B10-induced lysosomal permeabilization and cell death (Supplementary Figure S3). Because $\alpha$-tocopherol indirectly stimulates PI3K signaling and can exert additional
A

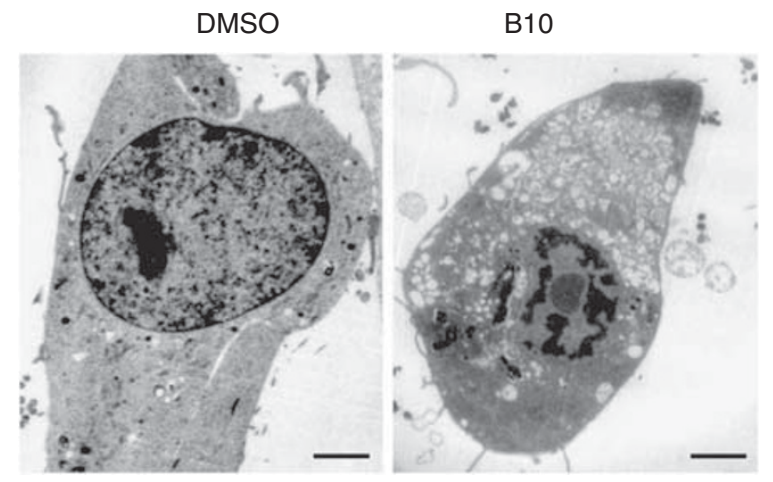

B

a

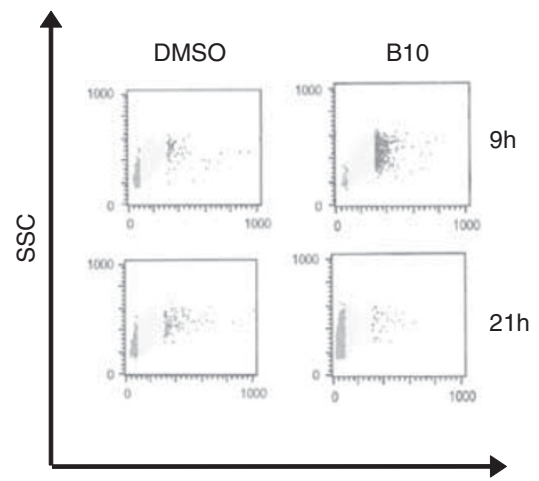

LTR (FL2)
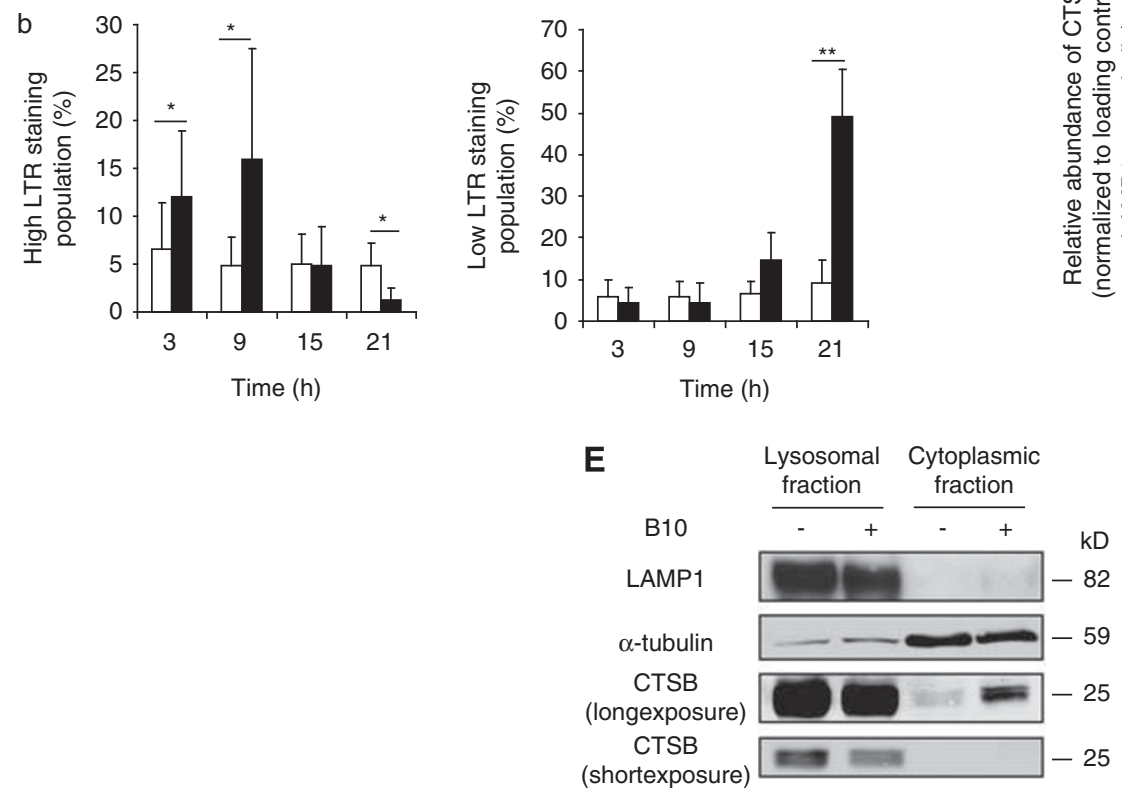

C

Phase
Contrast

CTSZ
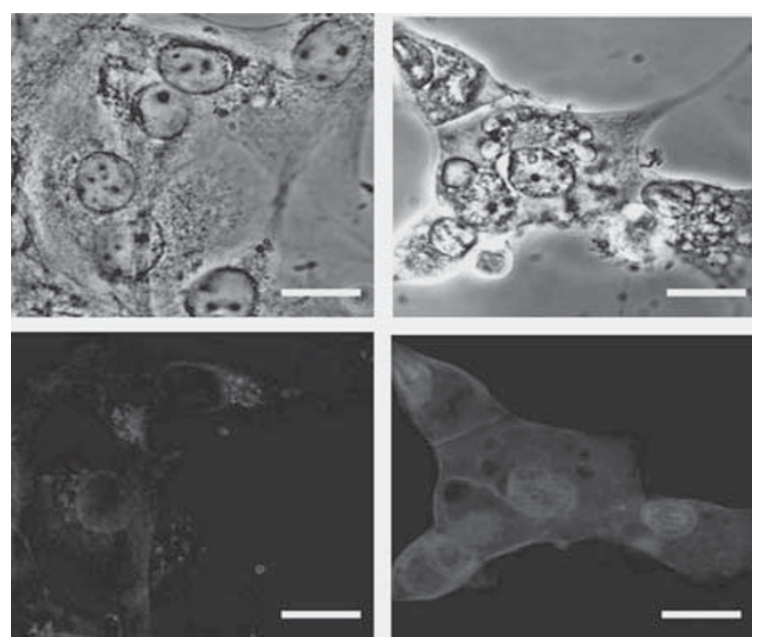

D
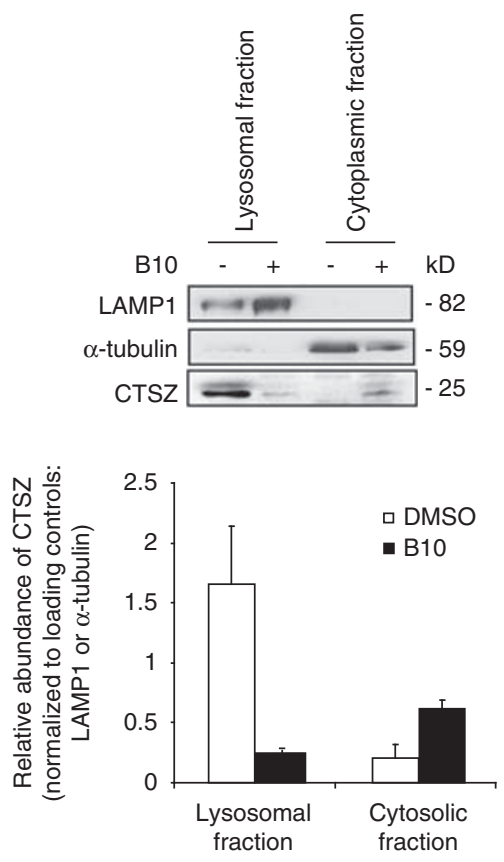
functions, for example, antioxidant functions, ${ }^{16}$ we also knocked down TSC2 to activate mTOR. However, silencing of TSC2 had no effect on mTOR signaling in glioblastoma cells and did not rescue the B10-induced LC3 conversion or cytotoxicity (Supplementary Figure S4). Therefore, additional studies are required to define the role of PI3K/Akt/mTOR signaling in B10-triggered autophagy.

Next, the integrity of the autophagic flux was evaluated, because autophagy relies on lysosomes for the degradation of autophagosomal cargos. To this purpose, LC3-II levels were assayed upon B10 treatment in the absence or presence of bafilomycin A1 (BafA1), a drug that blocks the fusion of autophagosomes with lysosomes. An increase of LC3-II was observed after $3 \mathrm{~h}$ in cells treated with $\mathrm{B} 10$ and BafA1 compared with B10 alone (Figure 4D). Moreover, LC3 lipidation at $3 \mathrm{~h}$ was increased in B10-treated cells in the presence of BafA1 compared with cells treated with BafA1 alone (Figure 4D). These findings indicate that B10 induces autophagy at an early time point, while LC3-II levels in B10treated cells in the presence of BafA1 were similar to cells treated with BafA1 alone at later time points (Figure 4D), suggesting that the autophagic flux is progressively disrupted

A

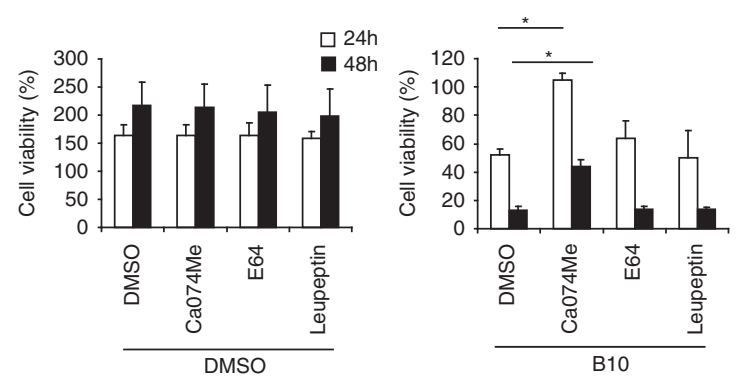

B
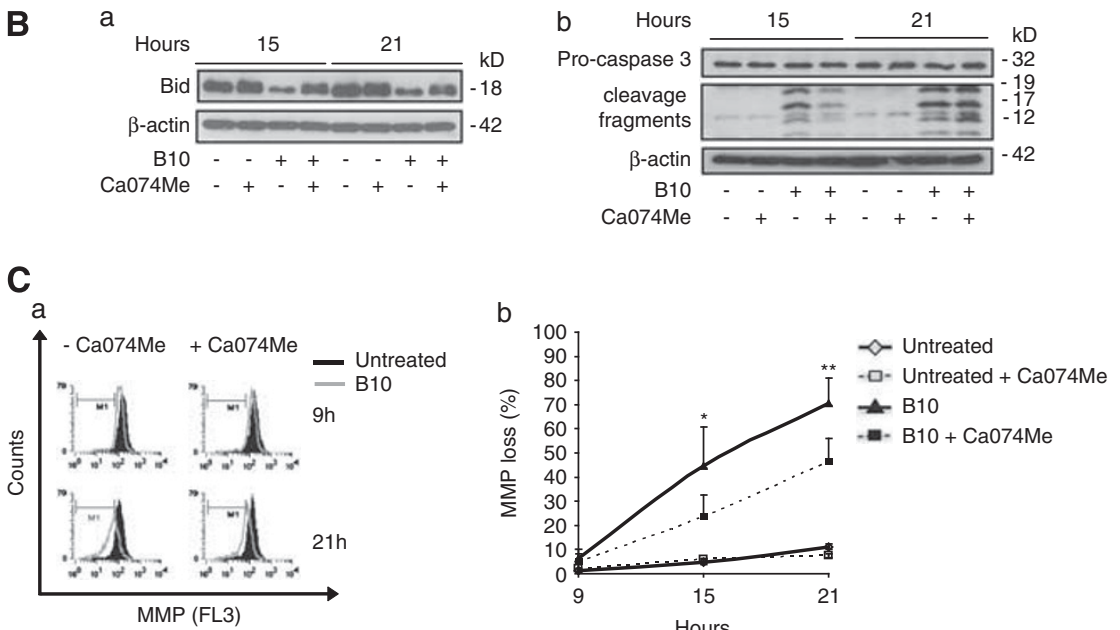

Figure 3 Ca074Me delays B10-induced cell death and transiently reduces Bid degradation, caspase-3 activation and mitochondrial membrane depolarization. (A) U87MG cells were incubated with $18 \mu \mathrm{M} \mathrm{B10}$ or DMSO in combination with Ca074Me $(10 \mu \mathrm{M}), \mathrm{E} 64(50 \mu \mathrm{M})$ or leupeptin $(100 \mu \mathrm{M})$. Cell viability was quantified by crystal violet staining after $24 \mathrm{~h}$ (black bars) and $48 \mathrm{~h}$ (white bars). Results are expressed as the percentage of cell density value prior to stimulation. Mean + S.D. of three independent experiments are shown, ${ }^{*} P<0.01$. (B) Expression levels of Bid (a) and caspase-3 cleavage (b) were analyzed by immunoblot after $15 \mathrm{~h}$ and $21 \mathrm{~h}$ incubation with $18 \mu \mathrm{M} \mathrm{B10}$ or DMSO in the presence or absence of $10 \mu \mathrm{M} \mathrm{Ca074Me.} \mathrm{(C)} \mathrm{Mitochondrial} \mathrm{membrane} \mathrm{potential} \mathrm{was} \mathrm{measured} \mathrm{by} \mathrm{flow} \mathrm{cytometry} \mathrm{and} \mathrm{TMRM} \mathrm{staining} \mathrm{of} \mathrm{cells} \mathrm{treated} \mathrm{for} \mathrm{indicated}$ times with $18 \mu \mathrm{M} \mathrm{B10}$ or DMSO in the presence or absence of Ca074Me. (a) Representative histograms are shown. (b) Quantification of the percentage of cells with reduced MMP is represented. Mean + S.D. of four independent experiments are shown; ${ }^{\star} P<0.02,{ }^{\star \star} P<0.05$ comparing cells treated with B10 and Ca074Me versus cells treated with $\mathrm{B} 10$ alone

Figure 2 B10 destabilizes the lysosomal compartment. (A) Electron microscopy was performed on U87MG cells treated with $18 \mu \mathrm{M}$ B10 or DMSO for $24 \mathrm{~h}$. Representative pictures are presented, scale bar: $3000 \mathrm{~nm}$. (B) The volume of the acidic compartments B10- or DMSO-treated cells was analyzed by LTR staining and flow cytometry. (a) Representative dot plots are shown at $9 \mathrm{~h}$ and $21 \mathrm{~h}$, coloring of the dots distinguishes populations of cells according to their fluorescence intensity. Red, yellow and green colors stand for populations of cells with high, normal and low LTR fluorescence intensity, respectively. (b) Quantitative analysis of cell populations with high and low LTR fluorescence intensity of cells treated with $18 \mu \mathrm{M} \mathrm{B10}$ (black bars) or DMSO (white bars). Mean + S.D. of six independent experiments are shown, ${ }^{*} P<0.04$; ${ }^{* \star} P<0.01$. (C) CTSZ staining of cells treated with $18 \mu \mathrm{M} \mathrm{B10}$ or DMSO for $24 \mathrm{~h}$ was performed and analyzed by confocal microscopy (scale bar: $20 \mu \mathrm{m}$ ). (D and E) Cell fractionation was performed to separate lysosomal and cytosolic fraction from DMSO- or B10-treated cells (21 h). CTSZ and CTSB were detected by western blotting in the different fractions. A representative blot is shown ( $\mathbf{D}$, upper panel and $\mathbf{E}$ ). The relative amounts of CTSZ were quantified from two independent experiments by normalizing CTSZ expression to LAMP1 or $\alpha$-Tubulin expression for the lysosomal and cytosolic fractions, respectively (D, lower panel). The color reproduction of this figure is available at the Cell Death and Differentiation journal online 
in B10-treated cells. In line with this notion, the levels of p62, a protein that is normally degraded upon fusion of autophagosomes with lysosomes, ${ }^{17}$ increased at later time points of B10 treatment (Figure 4E). Together, these observations indicate that $\mathrm{B} 10$ triggers both autophagy induction and progressively disrupts the autophagic flux leading to an accumulation of the undigested autophagosomes.

Destructive autophagy contributes to cell death upon B10 treatment. Autophagy can favor cell survival or on the
A

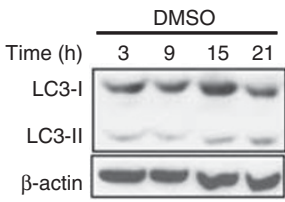

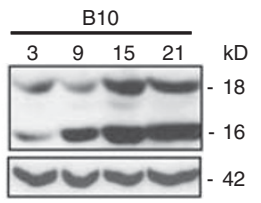

B

DMSO

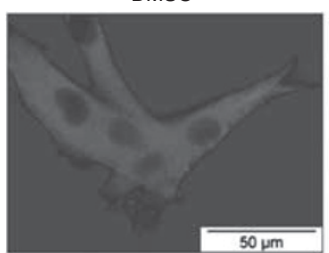

$50 \mu \mathrm{m}$
B10

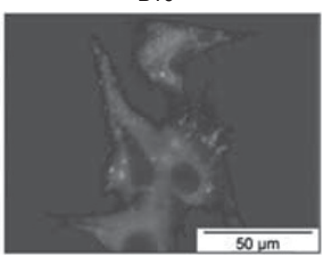

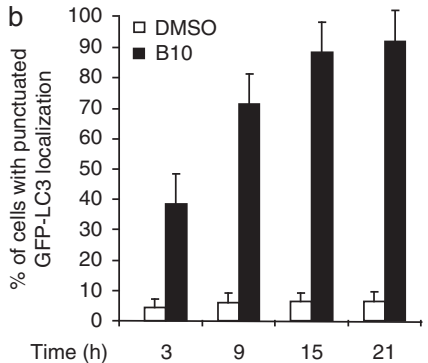

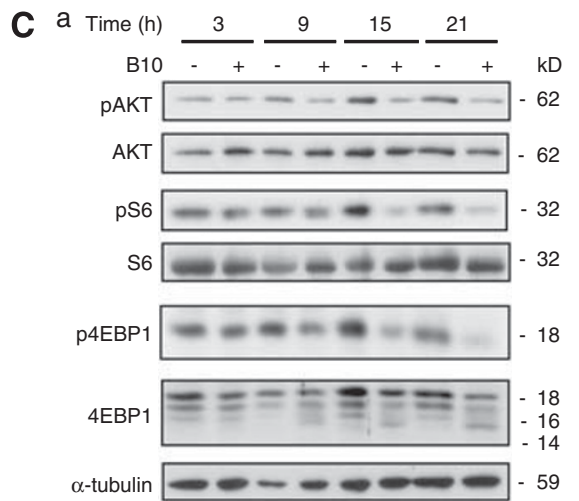

b
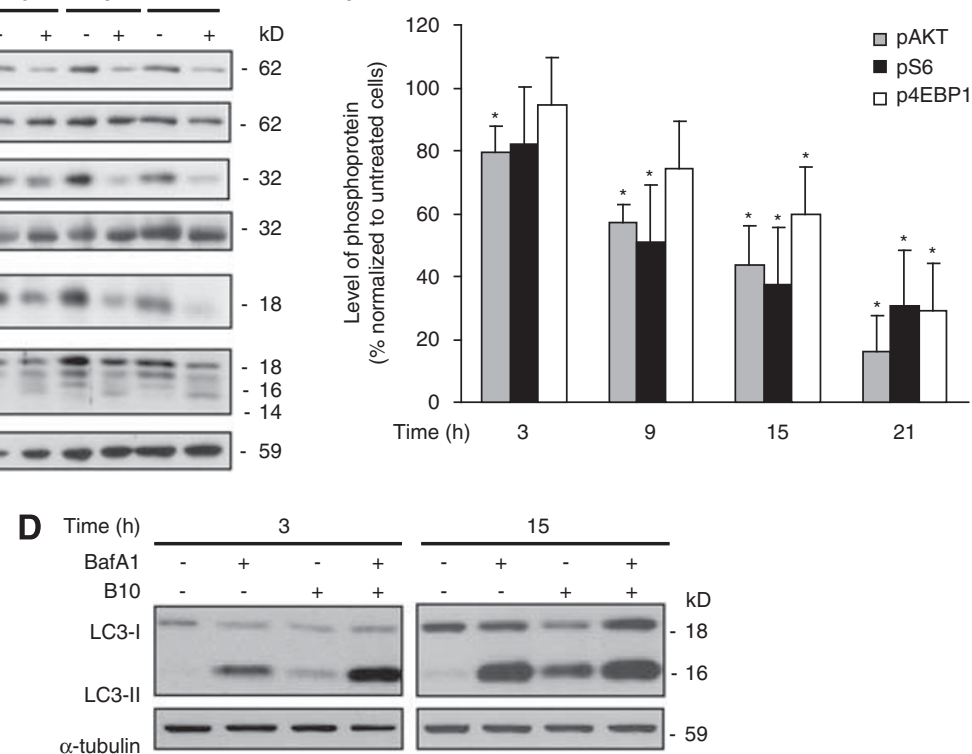

E

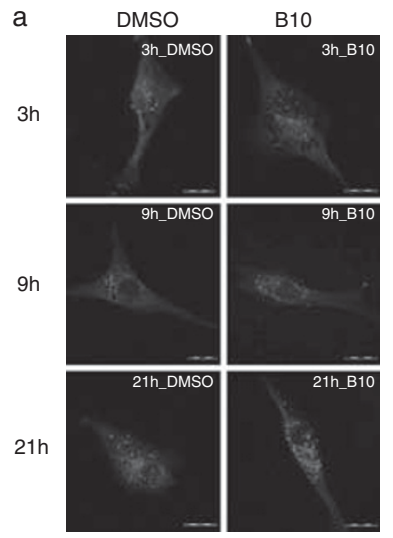

b

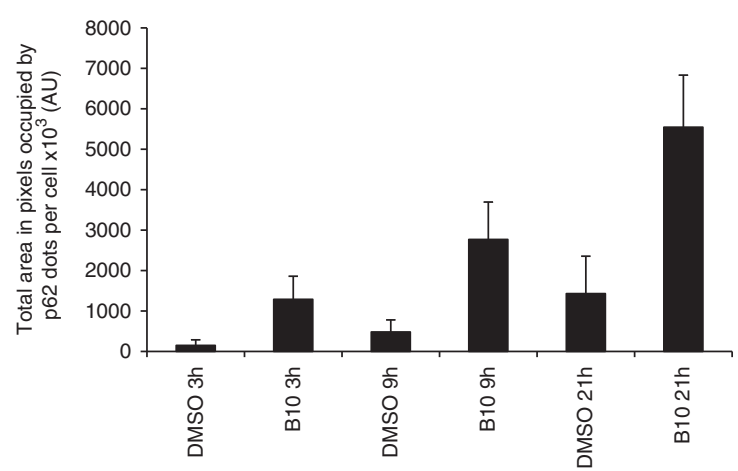


contrary can promote cell death under stress conditions. ${ }^{18,19}$ Importantly, PI3K/Akt/mTOR inhibition per se is not cytotoxic in U87MG cells as we and others previously reported, ${ }^{20,21}$ but recent evidence indicates that late-stage disruption of the autophagic process can cause detrimental autophagy. ${ }^{21}$
Therefore, the role of autophagy in B10-induced cell death was addressed by RNAi-mediated knockdown of ATG7, ATG5 or BECN1, key regulators of autophagy. C2-Ceramide was used as a positive control to induce autophagy. ${ }^{22}$ Downregulation of ATG7, ATG5 or BECN1 reduced a

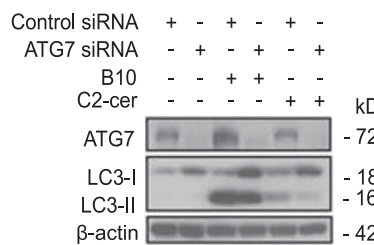

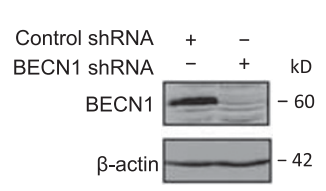

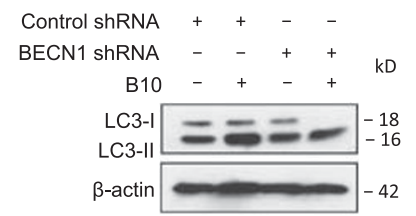

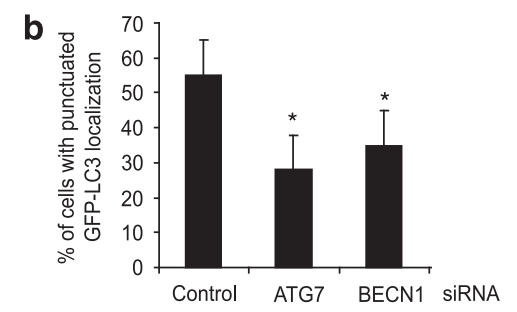

C
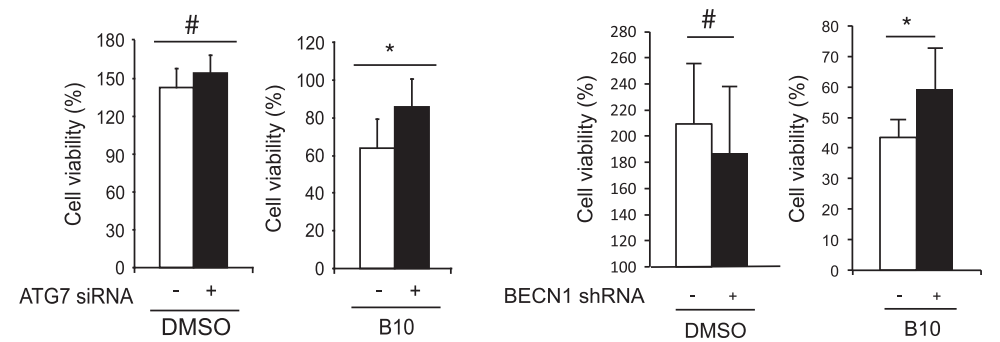

d
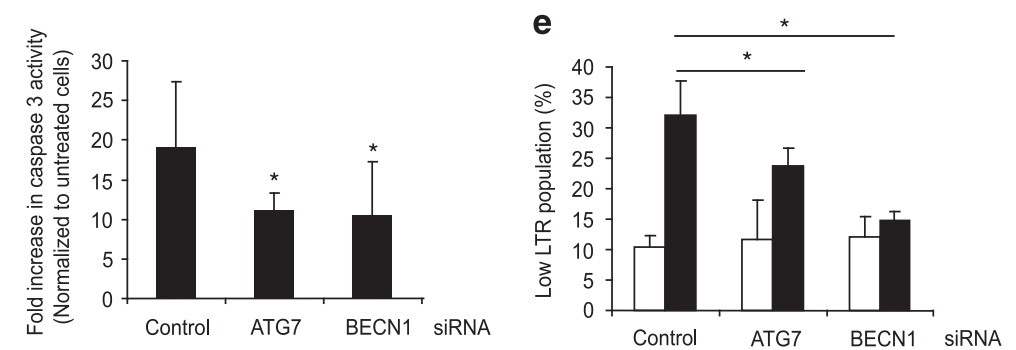

Figure 5 Autophagy promotes B10 cytotoxic activity. (a) U87MG cells transfected with ATG7 or control siRNA or with BECN1 or control shRNA vectors were treated with $18 \mu \mathrm{M} \mathrm{B10}$, DMSO or (C2-ceramide, $75 \mu \mathrm{M}$ ). LC3 lipidation was analyzed by immunoblot. (b) The amount of cells presenting a punctuated localization of GFP-LC3 was quantified upon treatment with $18 \mu \mathrm{M}$ B10. Mean + S.D. of three independent experiments are shown; ${ }^{*} P<0.05$. (c) Cell viability was determined by crystal violet staining in control cells (white bars) or ATG7 or BECN1 knockdown cells (black bars) after treatment for $24 \mathrm{~h}$ with $18 \mu \mathrm{M} \mathrm{B10}$ or DMSO. Mean + S.D. of at least three independent experiments are presented; ${ }^{*} P<0.05, \# P>0.05$. ( $\mathbf{d}$ and $\mathbf{e}$ ) The effect of ATG7 and BECN1 downregulation on caspase-3 activity using a caspase-3 fluorogenic substrate (d) or on lysosomal permeabilization using LTR staining (e) following treatment for $21 \mathrm{~h}$ with $18 \mu \mathrm{M} \mathrm{B10}$ (black bars) or without B10 (white bars) was analyzed. Mean + S.D. of three independent experiments are shown, ${ }^{*} P<0.05$

Figure 4 Accumulation of lipidated LC3 correlates with the PI3K/Akt/mTOR pathway inactivation upon B10 treatment. (A) U87MG cells were treated for indicated times with $18 \mu \mathrm{M} \mathrm{B10}$ or DMSO, and LC3 and its lipidated form were detected by immunoblot analysis. (B) The localization of endogenous LC3 was determined by immunofluorescence in cells treated with $18 \mu \mathrm{M} \mathrm{B10}$ or DMSO for $21 \mathrm{~h}(\mathrm{a}$ and $\mathrm{b})$. The kinetic of GFP-LC3 translocation was determined by counting cells presenting with a punctuated GFP-LC3 localization after treatment with $18 \mu \mathrm{M} \mathrm{B10}$ for indicated times. Mean + S.D. of three independent experiments are shown. (C) Phosphorylation status of Akt, ribosomal protein S6 and 4EBP1 was analyzed by western blot analysis upon with $18 \mu \mathrm{M} \mathrm{B10}$ or DMSO for the indicated time points (a). Quantitation of phosphoproteins was normalized to total protein levels and and mean + S.D. of three independent experiments are shown; $P$ values were calculated against DMSO, ${ }^{*} P<0.05$ (b). (D) U87MG cells were treated for indicated times with $18 \mu \mathrm{M} \mathrm{B10}$ or DMSO in the absence or presence of BafA1. LC3 and its lipidated form were detected by immunoblot analysis. (E) p62 protein expression was assessed by immunofluorescence using confocal microscopy in U87MG cells treated with $18 \mu \mathrm{M} \mathrm{B10}$ for indicated times; scale bar: $100 \mu \mathrm{m}$ (a). The area occupied by p62 dots per cell was quantified using ImageJ digital imaging software (b). Mean + S.D. of five cells per sample are shown 
B10-induced LC3 lipidation (Figure 5a, Supplementary Figure S5). Also, B10-mediated GFP-LC3 translocation to autophagosomes was significantly decreased upon ATG7 or BECN1 silencing (Figure 5b). Importantly, downregulation of ATG7, ATG5 or BECN1 significantly inhibited the B10-induced loss of cell viability (Figure 5c, Supplementary Figure S5), which argues for a detrimental role of autophagy in B10-induced cell death. Also, knockdown of ATG7 or BECN1 significantly reduced the B10-triggered increase in caspase-3-like activity (Figure $5 \mathrm{~d}$ ), indicating that autophagy at least partially contributes to caspase-3 activation. In addition, the effect of autophagy on lysosomal stability was evaluated, because accumulation of undigested autophagosomes might contribute to lysosomal permeabilization. ${ }^{23}$ ATG7 or BECN1 knockdown significantly suppressed B10triggered lysosomal permeabilization (Figure $5 \mathrm{e}$ ). This set of experiments indicates that B10-induced autophagy contributes to caspase-3 activation, lysosomal permeabilization and cell death. We also investigated the effect of combined inhibition of autophagy and lysosomal enzymes. Ca074Me cooperated with ATG7 knockdown to rescue cells from B10-induced loss of viability (Supplementary Figure S6), indicating that lysosomal disruption and autophagy contribute to cell death.

\section{Discussion}

In the present study, we identify a novel mode of action for the cytotoxic activity of the BA derivative B10 (Figure 6). This mechanism unexpectedly involves an early activation of autophagy and the permeabilization of lysosomes upon B10-treatment. This later event concomitantly leads to the release of lysosomal enzymes into the cytoplasm and impairs the late stages of autophagy, thus turning autophagy into a destructive process. Ultimately, these alterations commit cells to death via the apoptotic and non-apoptotic pathways.

B10-induced mixed phenotype of cell death is supported by the fact that DNA fragmentation does not closely correlate with cell death rates, that $\mathrm{Bcl}-2$ overexpression does not prevent nor delay B10-induced cell death and that downregulation of caspase-3 only partially rescues B10-induced cell death. In addition, we show that PI incorporation matches cell death kinetics, which points to necrotic features. Although the pancaspase inhibitor zVAD.fmk significantly delays cell death, this finding does not necessarily argue for caspase involvement in B10-induced cell death, because zVAD.fmk can also block other enzymes at the concentration used in this study, such as cathepsins, which have previously been reported to be involved in caspase-dependent and -independent cell death. ${ }^{13,24}$ Importantly, this might explain that apoptotic and non-apoptotic modes of cell death can coexist as a consequence of the intracytoplasmic release of lysosomal enzymes upon B10 treatment. Indeed, although cathepsin activity can lead to the activation of substrates specifically involved in apoptosis, such as Bid and caspase- $3,{ }^{12,14}$ their ability to cleave a large number of substrates can also commit cells to die via the non-apoptotic pathways. ${ }^{23,25}$ Accordingly, we observe that $\mathrm{Ca} 074 \mathrm{Me}$ only transiently reduces MMP loss and caspase- 3 activation. Firstly, this indicates that mitochondrial permeabilization also relies on lysosomal proteases

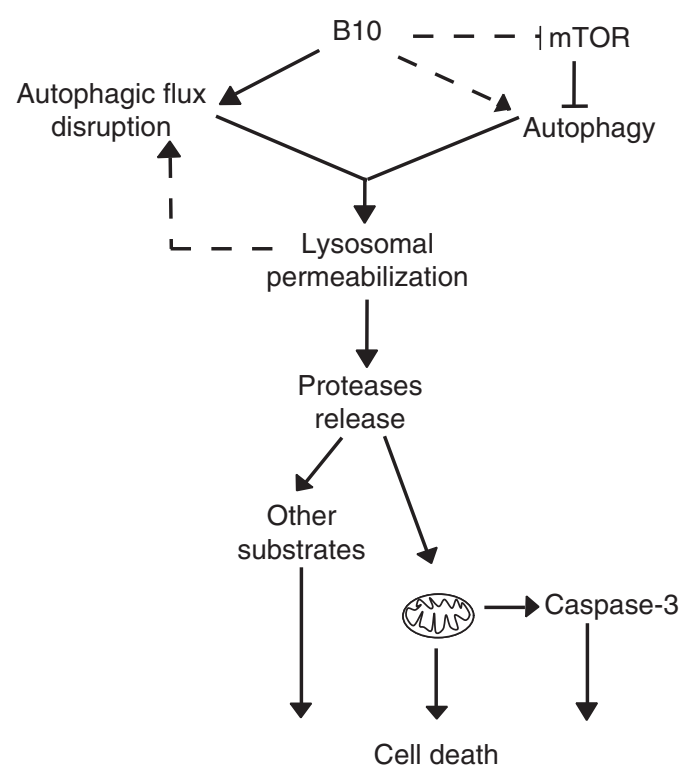

Figure 6 Proposed mode of action for B10 cytotoxic activity. B10 triggers autophagy in an early phase, either by Akt/mTOR inhibition and/or by the mTORindependent pathways. Progressively, B10 impairs the autophagic flux possibly by destabilizing lysosomes. In turn, the accumulation of enlarged and unstable autophagolysomes or the accumulation of undegraded material promotes the release of lysosomal enzymes into the cytoplasm. Subsequently, lysosomal proteases contribute directly or indirectly to apoptosis by caspase-3 activation and to non-apoptotic cell death by a non-selective degradation of cellular substrates

that cannot be inhibited by CA074Me and/or on other parallel processes arising upon B10 treatment such as direct permeabilization of mitochondrial membrane by $\mathrm{B} 10 .^{6}$ Secondly, because $\mathrm{Ca} 074 \mathrm{Me}$ delays cell death for significantly longer periods than caspase-3 activation, these results also implicate that lysosomal permeabilization has a role in cell death independently of induction of apoptosis via the mitochondrial pathway in B10-treated cells. This is possibly related to the degradation of substrates not directly implicated in apoptosis, but required for the maintenance of cell viability as discussed above. Together, these novel findings suggest that B10 might overcome resistance to apoptosis by exploiting alternative modes of cell death.

The role of autophagy in cell death is controversial, as there is evidence for pro- and anti-survival functions of this process depending on the context. ${ }^{18,19}$ Importantly, autophagy contributes to B10-induced cell death as silencing of autophagy genes, such as ATG5, ATG7 or BECN1, reduces cell death following treatment with B10. Although induction of autophagy is required to promote cell death upon B10 treatment, this is per se insufficient for cell death execution. This assumption is supported by the fact that PI3K/Akt/mTOR inhibition is not cytotoxic in U87MG, although it enhances autophagy (data not shown and Opel et $a l^{20}$; Degtyarev et $a l^{21}$ ). Therefore, a second event might be necessary to drive autophagy into its detrimental branch. Along this line, recent studies show that disruption of the late stages of autophagy can turn autophagy into a destructive process, ${ }^{21,26,27}$ suggesting that the conversion of protective autophagy into destructive autophagy might rely on a balance between autophagosome production and appropriate degradation capacity by the lysosomes. Remarkably, 
we demonstrate for the first time that the same conversion of the autophagic function is achieved upon single treatment with B10 in a sequence of two steps. Firstly, B10 induces autophagy and secondly, B10 disrupts the autophagic flux subsequently to lysosomal permeabilization. Although the exact underlying causes of B10-stimulated lysosomal permeabilization have not yet been determined, this might involve ROS generation and lipid peroxidation, because BA has previously been reported to trigger ROS production, ${ }^{6}$ or alternatively alterations in the metabolism of sterols, which are critical for the stability of biomembranes, as recently shown for another BA derivative. ${ }^{10}$ Disruption of the autophagic flux can lead to the excessive accumulation of autophagosomes containing deleterious undegraded material. ${ }^{21}$ Alternatively, a blockade of the fission step upon autophagolysosome formation might lead to the accumulation of enlarged and unstable acidic vesicles. The observation of large vesicles in B10-treated cells supports this latter hypothesis. In that case, autophagy would contribute to Iysosomal permeabilization by promoting cathepsin release from destabilized autophagolysosomes. ${ }^{28}$ The question whether these events are indeed key mediators in B10-induced cell death deserves further investigation.

In summary, we report that B10-induced cell death involves induction of autophagy and lysosomal permeabilization that turns autophagy into a cell death process and leads to the release of lysosomal enzymes into the cytoplasm. These novel properties of $\mathrm{B} 10$ are expected to have important implications for the therapeutic exploitation of BA derivatives, particularly in apoptosis-resistant cancers.

\section{Materials and Methods}

Cell culture and reagents. Cell lines were maintained at $37^{\circ} \mathrm{C}$ under $5 \%$ $\mathrm{CO}_{2}$ in DMEM medium Life Technologies Inc. (Eggenstein, Germany) supplemented with $10 \%$ FBS (Life Technologies $\mathrm{Inc}$.), $1 \mathrm{mmol} / \mathrm{l}$ glutamine (Biochrom, Berlin, Germany), 1\% penicillin/streptomycin (Biochrom) and $25 \mathrm{mmol} / \mathrm{l} \mathrm{HEPES}$ (Biochrom). B10 was synthesized by BioService Halle (Halle, Germany). Ca074Me, E64, leupeptin and $\alpha$-tocopherol were purchased from Sigma (Taufkirchen, Germany) and zVAD.fmk from Bachem (Weil am Rhein, Germany).

Determination of cell viability. Cell viability was evaluated by $3-(4,5-$ dimethylthiazol-2-yl)-2,5-diphenyltetrazolium bromide (MTT) assay (Roche Diagnostics, Mannheim, Germany) as described ${ }^{29}$ or by measuring cell density using crystal violet staining. Briefly, cells were fixed and stained for $10 \mathrm{~min}$ at room temperature (RT) by crystal violet solution $(0.5 \%$ of crystal violet, $30 \%$ of ethanol and $3 \%$ of paraformaldehyde). Plates were then washed in water and crystal violet incorporated by the cells was resolubilised in a solution containing $1 \%$ SDS Absorbance at $550 \mathrm{~nm}$ was measured using a microplate reader (EL800, Biotek, Bad Friedrichshall, Germany). All experiments are expressed as percentage of the value of cell density measured prior to stimulation. When indicated, zVAD.fmk, E64, Ca074Me, leupeptin or $\alpha$-tocopherol were used at $40 \mu \mathrm{M}, 50 \mu \mathrm{M}, 10 \mu \mathrm{M}, 100 \mu \mathrm{M}$ and $500 \mu \mathrm{M}$, respectively.

Determination of apoptosis. Fluorescence-activated cell-sorting (FACScan, Becton Dickinson, Heidelberg, Germany) analysis of DNA fragmentation of PIstained nuclei was performed as described. ${ }^{30}$ Annexin V/PI (BD Biosciences, Heidelberg, Germany) staining was performed according to the manufacturer's protocol. Caspase-3-like activity was measured by FACS (FL1) using the fluorogenic substrate $N$-benzyloxycarbonyl-Asp-Glu-Val-Asp-fluoromethylketone-R110 (zDEVDR110, Molecular Probes, Eugene, OR, USA) conjugated to rhodamine R110 that measures the activities of caspase-3 and other DEVD-specific proteases such as caspase-7. U87MG cells stably overexpressing Bcl-2 were obtained by transducing cells with a virus containing empty pMSCV vector or pMSCV vector encoding murine Bcl-2. Cells were selected with $10 \mu \mathrm{g} / \mathrm{ml}$ of blasticidin.
Western blot analysis. Cells were lysed in a lysis buffer containing $30 \mathrm{mM}$ Tris, $150 \mathrm{mM} \mathrm{NaCl}, 1 \%$ Triton X-100 and $10 \%$ glycerol for $15 \mathrm{~min}$ on ice. A solution of $200 \mu \mathrm{M}$ phenylmethanesulfonyl fluoride (PMSF), $2 \mathrm{mM}$ dithiothreitol, $1 \mu \mathrm{M}$ sodiumorthovanadate, $1 \mathrm{mM} \beta$-Glycerophosphate and $5 \mathrm{mM} \mathrm{NaF}$ were added to the lysis buffer when samples were prepared for phosphoprotein detection. After centrifugation at 13000 r.p.m. for 15 min at $4{ }^{\circ} \mathrm{C}$, the supernatant was collected and protein levels were quantified. The samples were loaded onto an acrylamide gel and then transferred on a PVDF or nitrocellulose membrane. Immunodetection of proteins was performed using anti-caspase-3, anti-Bid, anti-phospho-Akt (Ser-473), anti-phospho-S6 ribosomal protein and anti-S6 ribosomal protein, all from Cell Signaling (Beverly, MA, USA), anti-Akt (BD Biosciences), anti-LC3 (Thermo Fisher Scientific, Braunschweig, Germany), anti-Beclin1 (Santa Cruz Biotechnology, Santa Cruz, CA, USA), anti-ATG7 (Abcam, Cambridge, UK), anti-p62 (Sigma), antiCD107a (Lamp1) (BD Pharmingen, Heidelberg, Germany), anti-3-actin (Sigma) and anti- $\alpha$-Tubulin (Calbiochem, Darmstadt, Germany) antibodies. ImageJ (Wayne Rasband, NIH, Bethesda, MD, USA) was used to quantify protein levels. Expression of phosphoproteins was normalized to $\alpha$-Tubulin levels.

Determination of autophagy and autophagic flux integrity. GFPLC3-overexpressing U87MG cells were generated by selecting cells transfected with pBabe-GFP-LC3. ${ }^{31}$ Using an inverted fluorescence microscope (CK40, Olympus, Hamburg, Germany), the amount of cells presenting a punctuated localization for GFP-LC3 was determined after counting a minimum of 100 cells over at least three different fields of observation. Cut-off level for positivity was set to 1 puncta/cell for untransfected U87MG and to 5 puncta/cell for U87MG cells transfected with control, ATG7 or BECN1 small interfering RNA (siRNA). The data presented are the result from three independent experiments made in duplicates. To evaluate the status of the autophagic flux, $20 \mathrm{nM}$ of BafA1 was added to the culture medium $2 \mathrm{~h}$ before cell lysis in order to inhibit the degradation of autophagosomes by blocking their fusion with lysosomes. ${ }^{32}$ Lysates were analyzed by immunoblotting for LC3.

Immunofluorescence staining. Cells were fixed/permeabilized in a precooled methanol: acetone $(1: 1)$ solution for $10 \mathrm{~min}$ at $4^{\circ} \mathrm{C}$. Next, the slides were incubated in a PBS solution containing $0.1 \%$ of triton for $5 \mathrm{~min}$ at RT and washed with PBS before incubation overnight at $4{ }^{\circ} \mathrm{C}$ with a rabbit anti-LC3 antibody (Thermo Fisher Scientific) or a rabbit anti-CTSZ antibody (Abcam) diluted in PBS solution containing $2 \%$ BSA. The slides were then washed in PBS and incubated with an anti-rabbit lgG $(\mathrm{H}+\mathrm{L})$ antibody coupled to Texas-Red (Vector Laboratories Inc., Burlingame, CA, USA) or a sheep anti-mouse IgG antibody coupled to FITC (Millipore, Schwalbach, Germany) for $2 \mathrm{~h}$ at RT. Slides were imaged by an epifluorescence microscope (AX70, Olympus) for LC3 staining and images were analyzed by analySIS software (Olympus Soft Imaging System $\mathrm{GmbH}$ ). Fluoview FV10i confocal laser microscope (Olympus) was used to image CTSZ staining.

Cell fractionation. At $21 \mathrm{~h}$ after stimulation, 1.4 millions/sample were collected and washed twice in ice-cold extraction buffer $(210 \mathrm{mM}$ mannitol, $70 \mathrm{mM}$ sucrose, $1 \mathrm{mM}$ EDTA and $20 \mathrm{mM}$ Hepes, pH 7.5) complemented with $100 \mathrm{mM}$ PMSF and protease inhibitors (Roche Diagnostics). Cells were disrupted into the extraction buffer by passing the sample through a G27 needle until approximately $50 \%$ of the cells were positive for trypan blue. The total extracts were centrifuged at $1000 \mathrm{~g}$ for $5 \mathrm{~min}$ at $4{ }^{\circ} \mathrm{C}$. The supernatants were collected and centrifuged at $20800 \mathrm{~g}$ for $1 \mathrm{~h}$ at $4{ }^{\circ} \mathrm{C}$. The supernatant (cytosolic fraction) was collected and the pellet (lysosomal fraction) was lysed in the lysis buffer described in the procedure for western blotting. Samples were analyzed by immunoblotting.

Determination of mitochondrial membrane potential. To measure mitochondrial membrane potential, cells were incubated for $10 \mathrm{~min}$ with $100 \mathrm{ng} / \mathrm{ml}$ of tetramethylrhodamine methyl ester perchlorate (TMRM) (Sigma) at $37^{\circ} \mathrm{C}$, trypsinized, washed and immediately analyzed using flow cytometry.

Staining of the acidic compartment. Staining of the acidic compartment of cells was performed using LTR, a lysosomotropic probe (Invitrogen, Darmsadt, Germany). The cells were incubated for $10 \mathrm{~min}$ at $37^{\circ} \mathrm{C}$ with $50 \mathrm{nM}$ of LysoTracker in complete medium. Fluorescence intensity was then measured using a FACScalibur on channel 2 (FL2). The results were analyzed using Cell Quest (Becton Dickinson) by defining three cell populations determined by low, medium or high LTR fluorescence. Dead cells were excluded from the analysis by FSC/SSC analysis. 
Electron microscopy. Cells fixed with $2.5 \%$. glutaraldehyde, $0.1 \mathrm{M}$ phosphate cacodylate and $1 \%$ saccharose, $\mathrm{pH}$ 7.3. After dehydratation, thin layers were stained using osmium tetraoxyde. Imaging was performed using a Philips 400 Transmission electron microscope (Hamburg, Germany).

Downregulation of protein expression using RNA interference. Cells grown for $24 \mathrm{~h}$ were transfected with single-siRNA duplexes using transmessenger transfection reagent from Qiagen (Hilden, Germany). All siRNAs were purchased from Invitrogen (control siRNA: negative universal control 46-2001, caspase-3: HSS101372, Bid: BIDHSS141377 and ATG7: ATG7HSS116183). At $72 \mathrm{~h}$ post transfection, transfected cells were trypsinized and reseeded at equivalent cell density in each experiments. For gene silencing by lentiviral shRNA vectors, HEK293T cells were transfected with $7.5 \mu \mathrm{g}$ pGIPZ-shRNAmir vector, $12.5 \mu \mathrm{g} \mathrm{pCMV-dR8.91} \mathrm{and} 1 \mu \mathrm{g} \mathrm{pMD2.G} \mathrm{(Addgene} \mathrm{no.}$ 12259) using calcium phosphate transfection. All pGIPZ-shRNAmir-vectors were purchased from Thermo Fisher Scientific (non-silencing control: RHS4346, ShATG5: RHS4430-98715191, shBECN1: RHS4430-101032612, shTSC2 shRNA_1: RHS4430-99879929 and TSC2 shRNA_2: RHS4430-101130117). Virus containing supernatant was collected, filtered and used for spin transduction of U87MG cells at RT in the presence of $8 \mu \mathrm{g} / \mathrm{ml}$ polybrene. Transduced cells were selected with $1 \mu \mathrm{g} / \mathrm{ml}$ puromycin.

Statistical analysis. Statistical analysis was calculated using Student's t-test (two-tailed distribution, two-sample, unequal variance).

\section{Conflict of Interest}

The authors declare no conflict of interest.

Acknowledgements. We thank $\mathrm{C}$ Payer for expert technical assistance and C Hugenberg for excellent secretarial assistance. This work has been partially supported by Grants from the Deutsche Forschungsgemeinschaft, Bundesministerium für Bildung und Forschung, European Community (ApopTrain, APO-SYS) and IAP6/18 (to SF).

1. Cragg GM, Newman DJ. Plants as a source of anti-cancer agents. $J$ Ethnopharmacol 2005; 100: 72-79.

2. Alakurtti S, Makela T, Koskimies S, Yli-Kauhaluoma J. Pharmacological properties of the ubiquitous natural product betulin. Eur J Pharm Sci 2006; 29: 1-13.

3. Cichewicz RH, Kouzi SA. Chemistry, biological activity, and chemotherapeutic potential of betulinic acid for the prevention and treatment of cancer and HIV infection. Med Res Rev 2004; 24: 90-114.

4. Fulda $\mathrm{S}$, Kroemer G. Targeting mitochondrial apoptosis by betulinic acid in human cancers. Drug Discov Today 2009; 14: 885-890.

5. Chowdhury AR, Mandal S, Goswami A, Ghosh M, Mandal L, Chakraborty D et al. Dihydrobetulinic acid induces apoptosis in Leishmania donovani by targeting DNA topoisomerase I and II: implications in antileishmanial therapy. Mol Med 2003; 9: 26-36

6. Fulda S, Friesen C, Los M, Scaffidi C, Mier W, Benedict M et al. Betulinic acid triggers CD95 (APO-1/Fas)- and p53-independent apoptosis via activation of caspases in neuroectodermal tumors. Cancer Res 1997; 57: 4956-4964.

7. Kasperczyk H, La Ferla-Bruhl K, Westhoff MA, Behrend L, Zwacka RM, Debatin KM et al. Betulinic acid as new activator of NF-kappaB: molecular mechanisms and implications for cancer therapy. Oncogene 2005; 24: 6945-6956.

8. Qiu L, Wang Q, Di W, Jiang Q, Schefeller E, Derby S et al. Transient activation of EGFR/ AKT cell survival pathway and expression of survivin contribute to reduced sensitivity of human melanoma cells to betulinic acid. Int J Oncol 2005; 27: 823-830.
9. Rieber M, Rieber MS. Signalling responses linked to betulinic acid-induced apoptosis are antagonized by MEK inhibitor U0126 in adherent or 3D spheroid melanoma irrespective of p53 status. Int J Cancer 2006; 118: 1135-1143.

10. Willmann M, Wacheck V, Buckley J, Nagy K, Thalhammer J, Paschke R et al. Characterization of NVX-207, a novel betulinic acid-derived anti-cancer compound. Eur J Clin Invest 2009; 39: 384-394.

11. Fulda S, Meyer E, Debatin KM. Metabolic inhibitors sensitize for CD95 (APO-1/Fas)induced apoptosis by down-regulating Fas-associated death domain-like interleukin 1-converting enzyme inhibitory protein expression. Cancer Res 2000; 60: 3947-3956.

12. Droga-Mazovec G, Bojic L, Petelin A, Ivanova S, Romih R, Repnik U et al. Cysteine cathepsins trigger caspase-dependent cell death through cleavage of bid and antiapoptotic Bcl-2 homologues. J Biol Chem 2008; 283: 19140-19150.

13. Schotte $P$, Declercq W, Van Huffel S, Vandenabeele $P$, Beyaert R. Non-specific effects of methyl ketone peptide inhibitors of caspases. FEBS Lett 1999; 442: 117-121.

14. Stoka V, Turk B, Schendel SL, Kim TH, Cirman T, Snipas SJ et al. Lysosomal protease pathways to apoptosis. Cleavage of bid, not pro-caspases, is the most likely route. J Biol Chem 2001; 276: 3149-3157.

15. Vejux A, Guyot S, Montange T, Riedinger JM, Kahn E, Lizard G. Phospholipidosis and down-regulation of the PI3-K/PDK-1/Akt signalling pathway are vitamin $E$ inhibitable events associated with 7-ketocholesterol-induced apoptosis. J Nutr Biochem 2009; 20: 45-61.

16. Banks R, Speakman JR, Selman C. Vitamin E supplementation and mammalian lifespan. Mol Nutr Food Res 2010; 54: 719-725.

17. Bjorkoy G, Lamark T, Pankiv S, Overvatn A, Brech A, Johansen T. Monitoring autophagic degradation of p62/SQSTM1. Methods Enzymol 2009; 452: 181-197.

18. White E, DiPaola RS. The double-edged sword of autophagy modulation in cancer. Clin Cancer Res 2009; 15: 5308-5316.

19. Kenific CM, Thorburn A, Debnath J. Autophagy and metastasis: another double-edged sword. Curr Opin Cell Biol 2010; 22: 241-245.

20. Opel D, Westhoff MA, Bender A, Braun V, Debatin KM, Fulda S. Phosphatidylinositol 3-kinase inhibition broadly sensitizes glioblastoma cells to death receptor- and druginduced apoptosis. Cancer Res 2008; 68: 6271-6280.

21. Degtyarev M, De Maziere A, Orr C, Lin J, Lee BB, Tien JY et al. Akt inhibition promotes autophagy and sensitizes PTEN-null tumors to lysosomotropic agents. J Cell Biol 2008; 183: $101-116$

22. Scarlatti F, Bauvy C, Ventruti A, Sala G, Cluzeaud F, Vandewalle A et al. Ceramidemediated macroautophagy involves inhibition of protein kinase $B$ and up-regulation of beclin 1. J Biol Chem 2004; 279: 18384-18391.

23. Kagedal K, Zhao M, Svensson I, Brunk UT. Sphingosine-induced apoptosis is dependent on lysosomal proteases. Biochem J 2001; 359: 335-343.

24. Rozman-Pungercar J, Kopitar-Jerala N, Bogyo M, Turk D, Vasiljeva O, Stefe I et al. Inhibition of papain-like cysteine proteases and legumain by caspase-specific inhibitors: when reaction mechanism is more important than specificity. Cell Death Differ 2003; 10 : 881-888.

25. Boya P, Kroemer G. Lysosomal membrane permeabilization in cell death. Oncogene 2008; 27: $6434-6451$.

26. Boya P, Gonzalez-Polo RA, Casares N, Perfettini JL, Dessen P, Larochette N et al Inhibition of macroautophagy triggers apoptosis. Mol Cell Biol 2005; 25: 1025-1040.

27. Kanzawa T, Germano IM, Komata T, Ito H, Kondo Y, Kondo S. Role of autophagy in temozolomide-induced cytotoxicity for malignant glioma cells. Cell Death Differ 2004; 11: 448-457.

28. Mora R, Abschuetz A, Kees T, Dokic I, Joschko N, Kleber S et al. TNF-alpha- and TRAILresistant glioma cells undergo autophagy-dependent cell death induced by activated microglia. Glia 2009; 57: 561-581.

29. Denizot F, Lang R. Rapid colorimetric assay for cell growth and survival. Modifications to the tetrazolium dye procedure giving improved sensitivity and reliability. $J$ Immunol Methods 1986; 89: 271-277.

30. Vogler M, Durr K, Jovanovic M, Debatin KM, Fulda S. Regulation of TRAIL-induced apoptosis by XIAP in pancreatic carcinoma cells. Oncogene 2007; 26: 248-257.

31. Kabeya Y, Mizushima N, Ueno T, Yamamoto A, Kirisako T, Noda T et al. LC3, a mammalian homologue of yeast Apg8p, is localized in autophagosome membranes after processing. EMBO J 2000; 19: 5720-5728.

32. Yamamoto A, Tagawa Y, Yoshimori T, Moriyama Y, Masaki R, Tashiro Y. Bafilomycin A1 prevents maturation of autophagic vacuoles by inhibiting fusion between autophagosomes and lysosomes in rat hepatoma cell line, H-4-II-E cells. Cell Struct Funct 1998; 23: 33-42. 